EPJ manuscript No.

(will be inserted by the editor)

\title{
Improving the convergence of the chiral expansion for nuclear forces I: Peripheral phases
}

\author{
E. Epelbaum ${ }^{1}$ a, W. Glöckle ${ }^{1}$ b , and Ulf-G. Meißner ${ }^{2}$ c \\ 1 Ruhr-Universität Bochum, Institut für Theoretische Physik II, D-44870 Bochum, Germany \\ 2 Universität Bonn, Helmholtz-Institut für Strahlen- und Kernphysik (Theorie), Nußallee 14-16, D-53115 Bonn \\ Received: date / Revised version: date
}

\begin{abstract}
We study the two-pion exchange potential at next-to-next-to-leading order in chiral effective field theory. We propose a new cut-off scheme for the pion loop integrals based on spectral function regularization. We show that this method allows for a consistent implementation of constraints from pionnucleon scattering. It leads to a much improved description of the partial waves with angular momentum $l \geq 2$ as compared to the calculation utilizing dimensional regularization.
\end{abstract}

PACS. 13.75.Cs Nucleon-nucleon interactions - 21.30.-x Nuclear forces - 12.39.Fe Chiral Lagrangians

\section{Introduction}

Effective field theory (EFT) has become a standard tool for analyzing the chiral structure of Quantum Chromodynamics (QCD) at low energies, where the perturbative expansion in powers of the coupling constant cannot be used. The chiral symmetry of QCD is spontaneously broken and the corresponding Goldstone bosons can be identified with the pions, if one considers the two flavor sector of the up and down quarks as done here. It is a general property of Goldstone bosons that their interactions become weak for small momenta. Chiral Perturbation Theory (CHPT) is the effective field theory of the Standard Model which allows to describe the interactions of pions and between pions and matter fields (nucleons, $\rho$-mesons, $\Delta$-resonances, ...) in a systematic way. This is achieved via an expansion of scattering amplitudes and transition currents in powers of a low-momentum scale $Q$ associated with small external momenta and with the pion (light quark) mass. Here, small means with respect to the scale of chiral symmetry breaking of the order of $1 \mathrm{GeV}$. Pion loops are naturally incorporated and all corresponding ultraviolet divergences can be absorbed at each fixed order in the chiral expansion by counter terms of the most general chiral invariant Lagrangian.

This perturbative scheme works well in the pion and pion-nucleon sector, where the interaction vanishes for vanishing external momenta in the chiral limit, for some early reviews see e.g. 1, 2, 3 . The situation in the fewnucleon sector is much more complicated. The main difficulty in the direct application of the standard methods

\footnotetext{
a email: evgeni.epelbaum@tp2.ruhr-uni-bochum.de

b email: walter.gloeckle@tp2.ruhr-uni-bochum.de

c email: meissner@itkp.uni-bonn.de
}

of CHPT to the two-nucleon $(2 \mathrm{~N})$ system is due to the non-perturbative aspects of the problem, the unnaturally large S-wave scattering lengths and the shallow nuclear bound states. One possible way to deal with this difficulty has been suggested by Weinberg, who proposed to apply CHPT to the kernel of the corresponding integral equation for the scattering amplitude, which can be viewed as an effective nucleon-nucleon $(\mathrm{NN})$ potential [5,6]

The first quantitative realization of the above idea has been carried out by Ordóñez and co-workers, who derived an (energy-dependent) NN potential and performed a numerical analysis of the $2 \mathrm{~N}$ system [7. The energyindependent representation of the chiral NN potential, which can be applied much easier in few-nucleon calculations, has been derived in 8 9,10. At leading order $(\mathrm{LO})$ in the chiral expansion the potential is given by the well established one-pion exchange (OPE) and two contact forces without derivatives. At next-to-leading order (NLO) OPE gets renormalized and the leading two-pion exchange (TPE) diagrams as well as seven more contact operators appear. At NNLO, one has to include subleading TPE with one insertion of dimension two pion-nucleon vertices (the corresponding low-energy constants (LECs) are denoted by $c_{1,3,4}$, we adhere to the notation of Ref. 3] ). Notice that no new contact forces contribute at this order. While the pion exchanges are governed by the underlying chiral symmetry, the contact forces represent our ignorance of the short-range physics and are not restricted by chiral symmetry.

The LECs $c_{1,3,4}$ enter the expressions for the pionnucleon $(\pi \mathrm{N})$ scattering amplitude at subleading $\left(Q^{2}\right)$ and higher orders and thus can be determined from the $\pi \mathrm{N}$ data. From the $Q^{2}$ analysis [1] one gets: $c_{1}=-0.64, c_{3}=$ $-3.90, c_{4}=2.25$ (here and in what follows the values of 
E. Epelbaum et al.: Improving the convergence of the chiral expansion for nuclear forces I: Peripheral phases

the $c_{i}$ 's are given in $\mathrm{GeV}^{-1}$ ). The values obtained from various $Q^{3}$ analyses [11,12,13,14,15] are in the ranges: $c_{1}=-0.81 \ldots-1.53, c_{3}=-4.70 \ldots-6.19, c_{4}=3.25 \ldots 4.12$ These bands are also consistent with expectations from resonance saturation 12 . Notice that the numerical values of $c_{3}$ and $c_{4}$ at both orders $Q^{2}$ and $Q^{3}$ are quite large, which can be partially explained by the fact that the LECs $c_{3,4}$ are to a large extent saturated by the $\Delta$ excitation. This implies that a new and smaller scale, namely $m_{\Delta}-m \sim 293 \mathrm{MeV}$, enters the values of these constants in EFT without explicit $\Delta$, see [12. At fourth order, these LECs get modified by quark mass dependent contributions [16] and also are affected when electromagnetic corrections are included 17. These modifications go beyond the accuracy of the calculations performed here but should be kept in mind if one wants to go to higher orders and/or systematically includes isospin violation.

The large numerical values of the $c_{i}$ 's lead to dramatic consequences in few-nucleon systems 18. The resulting subleading TPE correction calculated using dimensional regularization [9] (or equivalent schemes) turns out to be very strong already at intermediate distances $r \sim 1-2$ fm. This could, in principle, be compensated by the corresponding contact terms. However, such a compensation at NNLO is only possible in low partial waves (i.e. in $\mathrm{S}$-, $\mathrm{P}$-waves as well as for $\epsilon_{1}$ ) since the contact terms do not contribute to $\mathrm{D}$ - and higher partial waves at this order. The D- and F-waves may therefore serve as a sensitive test of chiral TPE exchange, as suggested by Kaiser et al. in [9], since higher partial waves are strongly dominated by OPE and less sensitive to TPE (as it is known since long, for an early nonperturbative approach see 19]). The conventional scenario of nuclear forces represented by existing OBE models and various phenomenological potentials suggests that the D- and higher partial wave NN interactions are weak enough to be treated perturbatively, see 18. Clearly, in such a framework one can not describe the low partial waves that show strong nonperturbative effects. Under this assumption Kaiser et al. 9] applied chiral EFT to perform a parameter-free calculation for the neutron-proton $(n p) \mathrm{D}$ - and higher partial waves and found rather poor convergence of the chiral expansion already at surprisingly low energies. While the LO and NLO predictions, which correspond to OPE and to OPE accompanied by the leading TPE corrections, already agree reasonably well with the Nijmegen phase shift analysis 20 (NPSA), taking into account subleading TPE at NNLO governed by the LECs $c_{1,3,4}$ destroys that agreement and leads to strong deviations from the data for $E_{\text {lab }}>50$ $\mathrm{MeV}$ in D-waves and for $E_{\text {lab }}>150 \mathrm{MeV}$ in F-waves.

In 21] we have demonstrated that the NNLO potential allows for a good description of the NN data, which is also visibly improved compared to the NLO results. Contrary to Ref. 9], we did not perform a perturbative expansion of the $n p \mathrm{~T}$-matrix in high partial waves and calculated phase shifts by solving the Lippmann-Schwinger (LS) equation for the NN T-matrix. We found that taking the momentum space cut-off in the LS equation of the order of $1 \mathrm{GeV}$ allows for a satisfactory description of all par- tial waves simultaneously. With such a large value of the cut-off, the isoscalar central TPE potential becomes already so strongly attractive that unphysical deeply bound states appear in the $D$ - and in the lower partial waves. Note that although such deeply bound states do not influence NN observables at low energies, they might show up in another processes (like e.g. $N d[18]$ and $\pi d[22$ scattering). Since the potential is very strong (and attractive) and there are no counter terms according to the power counting, changing the value of the cut-off clearly leads to a strong variation of the $D$-wave phase shifts. Higher order counter terms are needed in order to reduce the cutoff dependence of these observables and thus the problem with the slow convergence of the chiral expansion remains.

Motivated by the known cancellation between the $\pi \pi$ and $\pi \rho$ exchanges, which has been observed in boson exchange models of the nuclear force, we have constructed in Ref. [18 the NNLO* version of the NN potential without spurious deeply bound states. To achieve that, we adopted values of the LECs $c_{3,4}$, which are much smaller in magnitude than the ones obtained from $\pi \mathrm{N}$ scattering and result from subtracting the $\Delta$-contribution and fine tuning to NN observables: $c_{3}=-1.15 \mathrm{GeV}^{-1}, c_{4}=1.20 \mathrm{GeV}^{-1}$. This also allowed for a fairly good description of the Dand higher partial waves. Accounting for subleading TPE leads to small corrections in most channels and the chiral expansion for NN scattering seems to converge. Certainly, the situation is still far from being satisfactory since the small values of LECs $c_{3,4}$ are not compatible with $\pi \mathrm{N}$ scattering. Notice that the large values of these LECs are also supported by recent determinations from $p p$ and $n p$ partial wave analysis performed by the Nijmegen group 23 . 24 .

In the present work we explain the origin of the above mentioned problems and present a way to improve the convergence of the chiral expansion for the NN interaction. It allows to use the large values of $c_{i}$ 's consistent with $\pi \mathrm{N}$ scattering. We argue that the unphysically strong attraction in the isoscalar central part of chiral TPE at NNLO resulting when calculated using dimensional (or equivalent) regularization is due to high-momentum components of exchanged pions, which cannot be properly treated in an EFT. Using a cut-off regularization instead of dimensional one and taking reasonable values for the momentum space cut-off allows to remove spurious shortdistance physics associated with high-momentum intermediate states and to greatly improve the convergence of the chiral expansion. A similar idea has already been used a long time ago in the analysis of the octet baryon masses and the pion-nucleon sigma term 25] and was recently applied to improve the convergence of the SU(3) baryon chiral perturbation theory $26,27,28$. A critical discussion about the use of cut-off schemes is provided in Ref. 29]. Notice further that a finite momentum-space cut-off in chiral loops has been used to derive the (energydependent) expressions for TPE in Ref. 7]. We also propose a simple and convenient way to derive analytic expressions for regularized TPE in the momentum space based on the spectral function representation. 
E. Epelbaum et al.: Improving the convergence of the chiral expansion for nuclear forces I: Peripheral phases

Our manuscript is organized as follows. In section 2 we describe our formalism and present the explicit expressions for regularized TPE. In section 3 we apply the formalism to $n p \mathrm{D}$ - and higher partial waves and compare the results with the ones obtained from dimensionally regularized expressions. The summary and conclusions are given in section 4

\section{Formalism}

Energy-independent expressions for the chiral TPE at NLO and NNLO have been derived using different formalisms in Refs. 8 9, 10. The corresponding diagrams are shown symbolically in Fig. 1 The last diagram in the first line (NLO) requires a special treatment in order to avoid double counting of the iterated OPE. Notice further that the first two graphs in the second line lead to vanishing contributions to the NN force. The explicit time-ordered expressions for the potential can be found in [10. While OPE is of the order $\left(Q / \Lambda_{\chi}\right)^{0}$, where $\Lambda_{\chi}$ refers to the chiral symmetry breaking scale of the order of the $\rho$-meson mass, TPE at NLO and NNLO provides corrections of orders $\left(Q / \Lambda_{\chi}\right)^{2}$ and $\left(Q / \Lambda_{\chi}\right)^{3}$, respectively. In the present work we will adopt the counting rule for the nucleon mass $m \gg \Lambda_{\chi}$, which is required for Weinberg's power counting to be consistent [5] 7]. Therefore, we do not need to include relativistic $1 / m$-corrections $\left(1 / m^{2}\right.$-corrections $)$ to TPE (OPE) at the order considered. Notice that in the one-nucleon sector $m$ is usually treated on a same footing as $\Lambda_{\chi}$. The same counting rule has also been used in Ref. 9. The TPE contributions at NLO are given by:

$$
\begin{aligned}
V^{\mathrm{NLO}}= & \frac{g_{A}^{2}}{\left(2 F_{\pi}\right)^{4}}\left(\boldsymbol{\tau}_{1} \cdot \boldsymbol{\tau}_{2}\right) \int \frac{d^{3} l}{(2 \pi)^{3}} \frac{\left(\mathbf{l}^{2}-\mathbf{q}^{2}\right)}{\omega_{+} \omega_{-}\left(\omega_{+}+\omega_{-}\right)} \\
& -\frac{1}{8\left(2 F_{\pi}\right)^{4}}\left(\boldsymbol{\tau}_{1} \cdot \boldsymbol{\tau}_{2}\right) \int \frac{d^{3} l}{(2 \pi)^{3}} \frac{\left(\omega_{+}-\omega_{-}\right)^{2}}{\omega_{+}+\omega_{-}} \frac{1}{\omega_{+} \omega_{-}} \\
& -\frac{g_{A}^{4}}{2\left(2 F_{\pi}\right)^{4}} \int \frac{d^{3} l}{(2 \pi)^{3}} \frac{\omega_{+}^{2}+\omega_{+} \omega_{-}+\omega_{-}^{2}}{\omega_{+}^{3} \omega_{-}^{3}\left(\omega_{+}+\omega_{-}\right)} \\
& \times\left\{\left(\boldsymbol{\tau}_{1} \cdot \boldsymbol{\tau}_{2}\right)\left(\mathbf{l}^{2}-\mathbf{q}^{2}\right)^{2}\right. \\
& \left.+6\left(\sigma_{2} \cdot[\mathbf{q} \times \mathbf{l}]\right)\left(\sigma_{1} \cdot[\mathbf{q} \times \mathbf{l}]\right)\right\} .
\end{aligned}
$$

where $\sigma_{i}$ and $\boldsymbol{\tau}_{i}$ are the spin- and isospin-matrices of the nucleon $i, \mathbf{q}$ is the nucleon momentum transfer and $\omega_{ \pm}=\sqrt{(\mathbf{q} \pm \mathbf{l})^{2}+4 M_{\pi}^{2}}$. The subleading TPE potential reads:

$$
\begin{aligned}
V^{\mathrm{NNLO}}= & \frac{3 g_{A}^{2}}{16 F_{\pi}^{4}} \int \frac{d^{3} l}{(2 \pi)^{3}} \frac{\mathbf{l}^{2}-\mathbf{q}^{2}}{\omega_{-}^{2} \omega_{+}^{2}} \\
& \times\left(8 c_{1} M_{\pi}^{2}+c_{3}\left(\mathbf{l}^{2}-\mathbf{q}^{2}\right)\right) \\
- & \frac{c_{4} g_{A}^{2}}{4 F_{\pi}^{4}}\left(\boldsymbol{\tau}_{1} \cdot \boldsymbol{\tau}_{2}\right) \int \frac{d^{3} l}{(2 \pi)^{3}} \frac{1}{\omega_{+}^{2} \omega_{-}^{2}} \\
& \times\left(\sigma_{2} \cdot[\mathbf{q} \times \mathbf{l}]\right) \times\left(\sigma_{1} \cdot[\mathbf{q} \times \mathbf{l}]\right) .
\end{aligned}
$$

We will show in the next section how these integrals can be evaluated using different regularization schemes.

\subsection{Dimensional versus cut-off regularization}

The integrals in Eqs. (2.1), (2.2) are ultraviolet divergent and thus need to be regularized. Applying dimensional regularization the TPE potential takes the form [9]:

$$
V_{\mathrm{DR}}=V_{\mathrm{DR}, \text { non }- \text { pol. }}+V_{\mathrm{DR}, \text { pol. }},
$$

where the non-polynomial and polynomial (in $\mathbf{q}$ ) parts at NLO $V_{\mathrm{DR}, \text { non-pol. }}^{\mathrm{NLO}}$ and $V_{\mathrm{DR}}^{\mathrm{NLO}}$ pol. , respectively, read:

$$
\begin{aligned}
V_{\mathrm{DR}, \text { non-pol. }}^{\mathrm{NLO}}= & -\frac{\boldsymbol{\tau}_{1} \cdot \boldsymbol{\tau}_{2}}{384 \pi^{2} F_{\pi}^{4}} L(q)\left\{4 M_{\pi}^{2}\left(5 g_{A}^{4}-4 g_{A}^{2}-1\right)\right. \\
+ & \left.q^{2}\left(23 g_{A}^{4}-10 g_{A}^{2}-1\right)+\frac{48 g_{A}^{4} M_{\pi}^{4}}{4 M_{\pi}^{2}+q^{2}}\right\} \\
- & \frac{3 g_{A}^{4}}{64 \pi^{2} F_{\pi}^{4}} L(q)\left[\left(\sigma_{1} \cdot \mathbf{q}\right)\left(\sigma_{2} \cdot \mathbf{q}\right)\right. \\
& \left.-\left(\sigma_{1} \cdot \sigma_{2}\right) q^{2}\right], \\
V_{\mathrm{DR}, \text { pol. }}^{\mathrm{NLO}}= & \left(S_{1}^{\mathrm{DR}}+S_{2}^{\mathrm{DR}} q^{2}\right)\left(\boldsymbol{\tau}_{1} \cdot \boldsymbol{\tau}_{2}\right) \\
& +S_{3}^{\mathrm{DR}}\left[\left(\sigma_{1} \cdot \mathbf{q}\right)\left(\sigma_{2} \cdot \mathbf{q}\right)-\left(\sigma_{1} \cdot \sigma_{2}\right) q^{2}\right] .
\end{aligned}
$$

Here, we have set $q \equiv|\mathbf{q}|$ and the logarithmic loop function $L(q)$ is given by

$$
L(q)=\frac{\omega}{q} \ln \frac{\omega+q}{2 M_{\pi}}, \quad \omega=\sqrt{q^{2}+4 M_{\pi}^{2}} .
$$

Further,

$$
\begin{aligned}
S_{1}^{\mathrm{DR}} & =\frac{1}{384 \pi^{2} F_{\pi}^{4}}\left\{-18 M_{\pi}^{2}\left(5 g_{A}^{4}-2 g_{A}^{2}\right) \ln \frac{M_{\pi}}{\mu}+\alpha_{1} M_{\pi}^{2}\right\}, \\
S_{2}^{\mathrm{DR}} & =\frac{1}{384 \pi^{2} F_{\pi}^{4}}\left\{\left(-23 g_{A}^{4}+10 g_{A}^{2}+1\right) \ln \frac{M_{\pi}}{\mu}+\alpha_{2}\right\}, \\
S_{3}^{\mathrm{DR}} & =-\frac{3 g_{A}^{4}}{64 \pi^{2} F_{\pi}^{4}}\left\{\ln \frac{M_{\pi}}{\mu}+\alpha_{3}\right\},
\end{aligned}
$$

where $\mu$ is the scale of dimensional regularization and the $\alpha_{i}$ are polynomials in $g_{A}$, whose precise form depends on the choice of subtraction. The non-polynomial part as well as all terms proportional to $\ln \left(M_{\pi} / \mu\right)$, which are due to logarithmic divergences in Eq. (2.1), are unique and do not depend on the choice of subtraction. The $\mu$-dependence of the $V_{\mathrm{DR}, \text { pol. }}^{\mathrm{NLO}}$ is compensated by the corresponding NLO counter terms of the form

$$
\begin{aligned}
V_{\mathrm{cont}}^{\mathrm{NLO}} & =C_{1}(\mu) M_{\pi}^{2}\left(\boldsymbol{\tau}_{1} \cdot \boldsymbol{\tau}_{2}\right)+C_{2}(\mu) q^{2}\left(\boldsymbol{\tau}_{1} \cdot \boldsymbol{\tau}_{2}\right) \\
& +C_{3}(\mu)\left(\sigma_{1} \cdot \mathbf{q}\right)\left(\sigma_{2} \cdot \mathbf{q}\right)+C_{4}(\mu) q^{2}\left(\sigma_{1} \cdot \sigma_{2}\right)
\end{aligned}
$$

and the resulting renormalized NN potential does not depend on $\mu$. Application of dimensional regularization to nuclear potentials is also discussed in [30]. 
NLO:

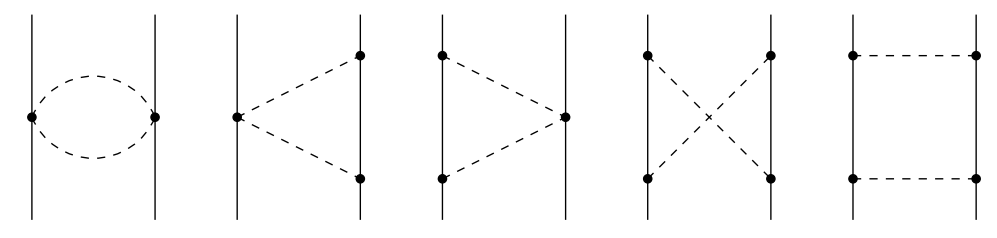

NNLO:

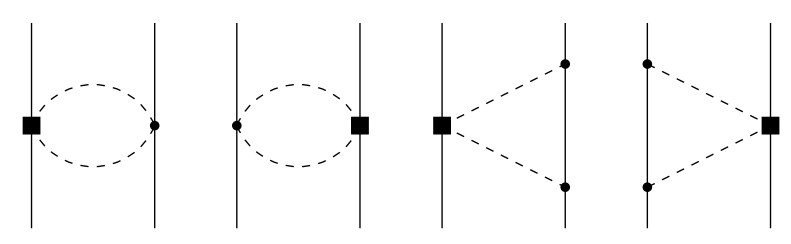

Fig. 1. Chiral TPE at NLO and NNLO. Heavy dots denote leading vertices from the chiral Lagrangian while solid rectangles correspond to subleading ones, which depend on the LECs $c_{1,3,4}$.

At NNLO one finds:

$$
\begin{aligned}
V_{\mathrm{DR}, \text { non-pol. }}^{\mathrm{NNLO}}=- & \frac{3 g_{A}^{2}}{16 \pi F_{\pi}^{4}}\left\{2 M_{\pi}^{2}\left(2 c_{1}-c_{3}\right)-c_{3} q^{2}\right\} \\
& \times\left(2 M_{\pi}^{2}+q^{2}\right) A(q) \\
& -\frac{g_{A}^{2}}{32 \pi F_{\pi}^{4}} c_{4}\left(4 M_{\pi}^{2}+q^{2}\right) A(q)\left(\boldsymbol{\tau}_{1} \cdot \boldsymbol{\tau}_{2}\right) \\
& \times\left[\left(\sigma_{1} \cdot \mathbf{q}\right)\left(\sigma_{2} \cdot \mathbf{q}\right)-q^{2}\left(\sigma_{1} \cdot \sigma_{2}\right)\right] \\
V_{\mathrm{DR}, \text { pol. }}^{\mathrm{NNLO}}= & \left(\tilde{S}_{1}^{\mathrm{DR}}+\tilde{S}_{2}^{\mathrm{DR}} q^{2}\right)+\tilde{S}_{3}^{\mathrm{DR}}\left(\boldsymbol{\tau}_{1} \cdot \boldsymbol{\tau}_{2}\right) \\
& \times\left[\left(\sigma_{1} \cdot \mathbf{q}\right)\left(\sigma_{2} \cdot \mathbf{q}\right)-\left(\sigma_{1} \cdot \sigma_{2}\right) q^{2}\right],
\end{aligned}
$$

with the loop function $A(q)$

$$
A(q)=\frac{1}{2 q} \arctan \frac{q}{2 M_{\pi}},
$$

and

$$
\begin{aligned}
\tilde{S}_{1}^{\mathrm{DR}} & =-\frac{3 g_{A}^{2}}{4 \pi F_{\pi}^{4}}\left(c_{1}-c_{3}\right) M_{\pi}^{3}, \\
\tilde{S}_{2}^{\mathrm{DR}} & =\frac{3 g_{A}^{2}}{16 \pi F_{\pi}^{4}} c_{3} M_{\pi}, \\
\tilde{S}_{3}^{\mathrm{DR}} & =-\frac{g_{A}^{2}}{32 \pi F_{\pi}^{4}} c_{4} M_{\pi} .
\end{aligned}
$$

Notice that the integrals in Eq. (2.2) are finite in dimensional regularization.

Although dimensional regularization provides an easy and convenient regularization scheme, it is by no means the only possible one. One can equally well regularize the divergent integrals in Eqs. (2.1), (2.2) using a momentum space cut-off, i.e. by multiplying the corresponding integrands by the regulating function $f_{\Lambda}(l) \equiv f_{\Lambda}(|\mathbf{l}|)$, with the properties $f_{\Lambda}(l) \stackrel{l \ll \Lambda}{\longrightarrow} 1, f_{\Lambda}(l) \stackrel{l \gg \Lambda}{\longrightarrow} 0$. This function $f_{\Lambda}(l)$ should go to 0 for large $l$ quick enough in order that the regularized integrals exist. For cut-off regularized (CR) TPE one finds similar to Eq. (2.3):

$$
V_{\mathrm{CR}}=V_{\mathrm{CR}, \text { non-pol. }}+V_{\mathrm{CR}, \text { pol. }},
$$

where

$$
\begin{aligned}
& V_{\mathrm{CR}, \text { non-pol. }}^{\mathrm{NLO}} \stackrel{\Lambda \rightarrow \infty}{\longrightarrow} V_{\mathrm{DR}, \text { non-pol. }}^{\mathrm{NLO}}, \\
& V_{\mathrm{CR}, \text { non-pol. }}^{\mathrm{NNLO}} \stackrel{\Lambda \rightarrow \infty}{\longrightarrow} V_{\mathrm{DR}, \text { non-pol. }}^{\mathrm{NNLO}} .
\end{aligned}
$$

The polynomial pieces have the same structure as in Eqs. (2.4), (2.8), where the quantities $S_{i}^{\mathrm{DR}}\left(\tilde{S}_{i}^{\mathrm{DR}}\right)$ should now be replaced by $S_{i}^{\mathrm{CR}}\left(\tilde{S}_{i}^{\mathrm{CR}}\right)$ given by

$$
\begin{aligned}
S_{1}^{\mathrm{CR}}= & \frac{1}{384 \pi^{2} F_{\pi}^{4}}\left\{-18 M_{\pi}^{2}\left(5 g_{A}^{4}-2 g_{A}^{2}\right) \ln \frac{M_{\pi}}{\Lambda}\right. \\
& \left.+\alpha_{1}^{\prime} M_{\pi}^{2}+\beta_{1} \Lambda^{2}\right\}, \\
S_{2}^{\mathrm{CR}}= & \frac{1}{384 \pi^{2} F_{\pi}^{4}}\left\{\left(-23 g_{A}^{4}+10 g_{A}^{2}+1\right) \ln \frac{M_{\pi}}{\Lambda}+\alpha_{2}^{\prime}\right\}, \\
S_{3}^{\mathrm{CR}}= & -\frac{3 g_{A}^{4}}{64 \pi^{2} F_{\pi}^{4}}\left\{\ln \frac{M_{\pi}}{\Lambda}+\alpha_{3}^{\prime}\right\},
\end{aligned}
$$

and

$$
\begin{aligned}
& \tilde{S}_{1}^{\mathrm{CR}}=-\frac{3 g_{A}^{2}}{4 \pi F_{\pi}^{4}}\left(\left(c_{1}-c_{3}\right) M_{\pi}^{3}+\beta_{2} M_{\pi}^{2} \Lambda+\beta_{3} \Lambda^{3}\right) \\
& \tilde{S}_{2}^{\mathrm{CR}}=\frac{3 g_{A}^{2}}{16 \pi F_{\pi}^{4}}\left(c_{3} M_{\pi}+\beta_{4} \Lambda\right), \\
& \tilde{S}_{3}^{\mathrm{CR}}=-\frac{g_{A}^{2}}{32 \pi F_{\pi}^{4}}\left(c_{4} M_{\pi}+\beta_{5} \Lambda\right) .
\end{aligned}
$$

Here $\alpha_{i}^{\prime}$ are polynomials in $g_{A}$ and $\beta_{i}$ are some combinations of $c_{i}$. The precise form of $\alpha_{i}^{\prime}, \beta_{i}$ depends on the choice of the regulating function $f_{\Lambda}(l)$. For a finite value of 


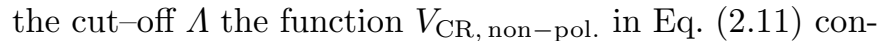
tains, in general, not only non-polynomial terms in $q$ but also polynomial ones, which are however suppressed by inverse powers of the cut-off $\Lambda$. The terms in Eqs. (2.13), (2.14) proportional to $\Lambda, \Lambda^{2}$ and $\Lambda^{3}$ correspond to linear, quadratic and cubic divergences in Eqs. (2.1), (2.2) and are absent in the dimensionally regularized expressions. Renormalization can be performed in very much the same way as before by absorbing the terms proportional to $\Lambda$, $\Lambda^{2}$ and $\ln \Lambda$ by the counter terms. The only difference is that we now need the $\mathrm{LO}$ counter terms in order to get rid of the $\Lambda^{2}$-term in Eq. (2.13) at NLO and the $\Lambda^{3}$-term in Eq. (2.14) at NNLO. In addition, NLO counter terms are required to renormalize the NNLO TPE potential. Notice that since $V_{\mathrm{CR}}$, non-pol. depends on $\Lambda$, the renormalized expressions for the potential using dimensional and cutoff regularizations are only identical with each other for $\Lambda \rightarrow \infty$. Does that mean that we should necessarily take $\Lambda \rightarrow \infty$ ? The answer is no. This is because in an EFT one is usually only able to calculate observables with a finite accuracy performing calculations up to a certain order in the low-momentum expansion. Taking $\Lambda \sim \Lambda_{\chi}$, the error from keeping $\Lambda$ finite is beyond the theoretical accuracy. In other words, since

$$
V_{\mathrm{CR}, \text { non-pol. }}=V_{\mathrm{DR}, \text { non-pol. }}+\mathcal{O}(1 / \Lambda)
$$

the DR and CR expressions are identical up to higher order terms. At NNLO one should choose $f_{\Lambda}(l)$ in such a way that $V_{\mathrm{CR}, \text { non-pol. }}^{\mathrm{NLO}}=V_{\mathrm{DR}, \text { non-pol. }}^{\mathrm{NLO}}+\mathcal{O}\left(1 / \Lambda^{2}\right)$.

\subsection{Spectral function representation}

Before discussing implications of the choice of the regularization scheme on the convergence of the chiral expansion, we will show how cut-off regularization of the potential can be understood in terms of the spectral function representation. By their very nature, these spectral functions are the most natural objects to separate the long- and short-distance contributions to the NN potential in momentum space. We will switch to the notation introduced in Ref. 9] and express $V^{\mathrm{NLO}}, V^{\mathrm{NNLO}}$ as:

$$
\begin{aligned}
V^{\mathrm{NLO}} & =W_{C}(q)\left(\boldsymbol{\tau}_{1} \cdot \boldsymbol{\tau}_{2}\right)+V_{S}(q)\left(\sigma_{1} \cdot \sigma_{2}\right) \\
& +V_{T}(q)\left(\sigma_{1} \cdot \mathbf{q}\right)\left(\sigma_{2} \cdot \mathbf{q}\right) \\
V^{\mathrm{NNLO}} & =V_{C}(q)+W_{S}(q)\left(\boldsymbol{\tau}_{1} \cdot \boldsymbol{\tau}_{2}\right)\left(\sigma_{1} \cdot \sigma_{2}\right) \\
& +W_{T}(q)\left(\boldsymbol{\tau}_{1} \cdot \boldsymbol{\tau}_{2}\right)\left(\sigma_{1} \cdot \mathbf{q}\right)\left(\sigma_{2} \cdot \mathbf{q}\right) .
\end{aligned}
$$

The functions $V_{i}(q)\left(W_{i}(q)\right)$ correspond to isoscalar (isovector) parts of the potential and can in case of dimensional regularization (and also for $\Lambda \rightarrow \infty$ ) be read off from Eqs. (2.4), (2.8). The subscripts $C, S$ and $T$ stand for the central, spin-spin and tensor contributions, in order.

The functions $V_{i}(q)\left(W_{i}(q)\right)$ can be represented (modulo terms polynomial in $\left.q^{2}\right)^{1}$ by a continuous superposi-

\footnotetext{
${ }^{1}$ In the present work we are only interested in the finiterange part of the two-pion exchange, which is given by the non-polynomial terms in momentum space.
}

tion of Yukawa functions 9, 31:

$$
\begin{aligned}
V_{i}(q) & =\frac{2}{\pi} \int_{2 M_{\pi}}^{\infty} d \mu \mu \frac{\rho_{i}(\mu)}{\mu^{2}+q^{2}}, \\
W_{i}(q) & =\frac{2}{\pi} \int_{2 M_{\pi}}^{\infty} d \mu \mu \frac{\eta_{i}(\mu)}{\mu^{2}+q^{2}},
\end{aligned}
$$

where $\rho_{i}(\mu)$ and $\eta_{i}(\mu)$ are the corresponding mass spectra (spectral functions). Note that subtracted dispersion integrals should be used in Eq. (2.17) if spectral functions do not decrease for large $\mu$. This usually happens in the EFT calculations, where the spectral functions are obtained within the low $-\mu$ expansion. Notice further that subtraction constants can be absorbed by the LECs corresponding to the short-range contact interactions and thus do not introduce any additional ambiguity. It is easy to see that $\rho_{i}(\mu)$ and $\eta_{i}(\mu)$ can be obtained from $V_{i}(q)$ and $W_{i}(q)$ via:

$$
\begin{aligned}
& \rho_{i}(\mu)=\operatorname{Im}\left[V_{i}\left(0^{+}-i \mu\right)\right], \\
& \eta_{i}(\mu)=\operatorname{Im}\left[W_{i}\left(0^{+}-i \mu\right)\right] .
\end{aligned}
$$

These spectral functions contain the whole dynamics corresponding to the exchanged $\pi \pi$ system. Once $\rho_{i}(\mu), \eta_{i}(\mu)$ functions are determined, the TPE potential can easily be obtained using Eq. (2.17).

Let us now calculate the spectral function $\rho_{C}^{\Lambda}(\mu)$, which results from the integral in Eq. (2.2) regularized with a cut-off $\Lambda$. We will choose the regulating function $f_{\Lambda}(l)$ as $f_{\Lambda}(l)=\theta(\Lambda-l)$. Performing integration over angles, one obtains

$$
\begin{aligned}
V_{C}^{\Lambda}(q)= & \frac{3 g_{A}^{2}}{128 \pi^{2} F_{\pi}^{4}} \int_{0}^{\Lambda} d l \frac{l\left(l^{2}-q^{2}\right)}{q\left(l^{2}+q^{2}+4 M_{\pi}^{2}\right)} \\
& \times\left(8 c_{1} M_{\pi}^{2}+c_{3}\left(l^{2}-q^{2}\right)\right) \\
& \times\left[\ln \left((l+q)^{2}+4 M_{\pi}^{2}\right)-\ln \left((l-q)^{2}+4 M_{\pi}^{2}\right)\right] .
\end{aligned}
$$

One then finds for the spectral function

$$
\begin{aligned}
\rho_{C}^{\Lambda}(\mu)= & \operatorname{Im}\left[V_{C}^{\Lambda}\left(0^{+}-i \mu\right)\right] \\
=- & \frac{3 g_{A}^{2}}{64 F_{\pi}^{4}}\left(2 M_{\pi}^{2}\left(2 c_{1}-c_{3}\right)+c_{3} \mu^{2}\right)\left(2 M_{\pi}^{2}-\mu^{2}\right) \\
& \times \frac{1}{\mu} \theta\left(\mu-2 M_{\pi}\right) \theta\left(\sqrt{\Lambda^{2}+4 M_{\pi}^{2}}-\mu\right) .(2.20)
\end{aligned}
$$

The entire $\Lambda$-dependence is contained in the second Heaviside step-function, which represents the only difference to the DR expression. Thus, as one could expect for physical reasons, cutting off the momentum $l$ of one of the exchanged pions at $l=\Lambda$ leads to a cut-off in the TPE spectral functions, which in this specific case takes the value $\sqrt{\Lambda^{2}+4 M_{\pi}^{2}} \sim \Lambda$. Clearly, the precise form of the resulting regulator in the spectral function representation depends on the choice of $f_{\Lambda}(l)$. Similar relations between 
the pion momentum and spectral function cut-offs can be obtained for other contributions to the NN potential as well. Using the regularized spectral function representation for TPE opens therefore an easy and convenient way to obtain the CR expressions and will be adopted in what follows. To be specific, we define the CR spectral functions $\rho_{i}^{\Lambda}(\mu), \eta_{i}^{\Lambda}(\mu)$ according to

$$
\begin{gathered}
\rho_{i}^{\Lambda}(\mu)=\rho_{i}(\mu) \theta(\Lambda-\mu), \\
\eta_{i}^{\Lambda}(\mu)=\eta_{i}(\mu) \theta(\Lambda-\mu),
\end{gathered}
$$

where $\rho_{i}(\mu), \eta_{i}(\mu)$ are the corresponding DR spectral functions, see section 2.3 The non-polynomial parts of the TPE potential at NLO and NNLO have then the same structure as in Eqs. (2.4), (2.8), where the loop functions $L(q)$ and $A(q)$ should be replaced by $L^{\Lambda}(q)$ and $A^{\Lambda}(q)$ defined as:

$$
\begin{aligned}
L^{\Lambda}(q) & =\theta\left(\Lambda-2 M_{\pi}\right) \frac{\omega}{2 q} \ln \frac{\Lambda^{2} \omega^{2}+q^{2} s^{2}+2 \Lambda q \omega s}{4 M_{\pi}^{2}\left(\Lambda^{2}+q^{2}\right)}, \\
s & =\sqrt{\Lambda^{2}-4 M_{\pi}^{2}} \\
A^{\Lambda}(q) & =\theta\left(\Lambda-2 M_{\pi}\right) \frac{1}{2 q} \arctan \frac{q\left(\Lambda-2 M_{\pi}\right)}{q^{2}+2 \Lambda M_{\pi}} .
\end{aligned}
$$

Several comments are in order. First of all we note that for $2 M_{\pi}<\Lambda$ the $\mathrm{CR}$ and $\mathrm{DR}$ expressions $V_{\mathrm{CR}}$ and $V_{\mathrm{DR}}$ only differ from each other by higher order contact interactions (i.e. by short-range terms) if $V_{\mathrm{CR}}$ is expanded in powers of $1 / \Lambda$. One can therefore use this regularization prescription in calculations at any given order in the lowmomentum expansion without getting into trouble with spurious long-range contributions suppressed by inverse powers of $\Lambda$ which might arise for a different choice of the cut-off function. Further, one should keep in mind that our choice of regularization is by no means unique. Different choices lead to equivalent results for the potential (up to higher order terms) and may be used as well. Finally, we would like to point out that the spectral function representation (2.17) does not allow to properly reproduce terms, which are polynomial in $q^{2}$ and non-analytic in $M_{\pi}^{2}$. Such terms are not important for our present work since we do not consider variation in $M_{\pi}$. If one is interested in the $M_{\pi}$-dependence of the nuclear force, see for instance Ref. 32, a cut-off regularization should be performed at the level of divergent integrals in Eqs. (2.1) and (2.2) rather than in the spectral function representation.

\subsection{Coordinate space representation}

The coordinate space representations $\tilde{V}_{C, S, T}(r)\left(\tilde{W}_{C, S, T}(r)\right)$ of the isoscalar (isovector) central, spin-spin and tensor parts of the potential $V_{C, S, T}(q)\left(W_{C, S, T}(q)\right)$ are defined according to

$$
\begin{aligned}
\tilde{V}(r)= & \tilde{V}_{C}(r)+\tilde{W}_{C}(r)\left(\boldsymbol{\tau}_{1} \cdot \boldsymbol{\tau}_{2}\right) \\
+ & \left(\tilde{V}_{S}(r)+\tilde{W}_{S}(r)\left(\boldsymbol{\tau}_{1} \cdot \boldsymbol{\tau}_{2}\right)\right)\left(\sigma_{1} \cdot \sigma_{2}\right) \\
+ & \left(\tilde{V}_{T}(r)+\tilde{W}_{T}(r)\left(\boldsymbol{\tau}_{1} \cdot \boldsymbol{\tau}_{2}\right)\right) \\
& \quad \times\left(3 \sigma_{1} \cdot \hat{r} \sigma_{2} \cdot \hat{r}-\sigma_{1} \cdot \sigma_{2}\right) .
\end{aligned}
$$

The functions $\tilde{V}_{C, S, T}(r)$ can be obtained for any given $r>0$ from the corresponding spectral functions via

$$
\begin{aligned}
& \tilde{V}_{C}(r)=\frac{1}{2 \pi^{2} r} \int_{2 M_{\pi}}^{\infty} d \mu \mu e^{-\mu r} \rho_{C}(\mu), \\
& \tilde{V}_{T}(r)=-\frac{1}{6 \pi^{2} r^{3}} \int_{2 M_{\pi}}^{\infty} d \mu \mu e^{-\mu r}\left(3+3 \mu r+\mu^{2} r^{2}\right) \rho_{T}(\mu), \\
& \tilde{V}_{S}(r)=-\frac{1}{6 \pi^{2} r} \int_{2 M_{\pi}}^{\infty} d \mu \mu e^{-\mu r}\left(\mu^{2} \rho_{T}(\mu)-3 \rho_{S}(\mu)\right) .
\end{aligned}
$$

The coordinate space representation of the isovector parts is given by the above equations replacing $\tilde{V}_{C, S, T}(r) \rightarrow$ $\tilde{W}_{C, S, T}(r)$ and $\rho_{C, S, T}(\mu) \rightarrow \eta_{C, S, T}(\mu)$.

We will now consider the coordinate space representation of the CR TPE potential. According to the definition (2.21), Eqs. (2.4), (2.8) and (2.18) we obtain for the CR spectral functions at NLO

$$
\begin{aligned}
\eta_{C}^{\Lambda}(\mu)= & \frac{1}{768 \pi F_{\pi}^{4}}\left\{4 M_{\pi}^{2}\left(5 g_{A}^{4}-4 g_{A}^{2}-1\right)\right. \\
& \left.-\mu^{2}\left(23 g_{A}^{4}-10 g_{A}^{2}-1\right)+\frac{48 g_{A}^{4} M_{\pi}^{4}}{4 M_{\pi}^{2}-\mu^{2}}\right\} \\
& \times \frac{\sqrt{\mu^{2}-4 M_{\pi}^{2}}}{\mu} \theta(\Lambda-\mu) \\
\rho_{T}^{\Lambda}(\mu)= & \frac{1}{\mu^{2}} \rho_{S}^{\Lambda}(\mu)=\frac{3 g_{A}^{4}}{128 \pi F_{\pi}^{4}} \frac{\sqrt{\mu^{2}-4 M_{\pi}^{2}}}{\mu} \theta(\Lambda-\mu),
\end{aligned}
$$

and at NNLO

$$
\begin{aligned}
& \rho_{C}^{\Lambda}(\mu)=- \frac{3 g_{A}^{2}}{64 F_{\pi}^{4}}\left(2 M_{\pi}^{2}\left(2 c_{1}-c_{3}\right)+c_{3} \mu^{2}\right) \\
& \times\left(2 M_{\pi}^{2}-\mu^{2}\right) \frac{1}{\mu} \theta\left(\mu-2 M_{\pi}\right) \theta(\Lambda-\mu) \\
& \eta_{T}^{\Lambda}(\mu)=\frac{1}{\mu^{2}} \eta_{S}^{\Lambda}(\mu)=-\frac{g_{A}^{2}}{128 F_{\pi}^{4}} c_{4}\left(4 M_{\pi}^{2}-\mu^{2}\right) \\
& \times \frac{1}{\mu} \theta\left(\mu-2 M_{\pi}\right) \theta(\Lambda-\mu) .
\end{aligned}
$$

We are now in the position to discuss implications of keeping the momentum space cut-off $\Lambda$ in the above expressions for the spectral functions finite. Let us consider, for example, the isoscalar central part of the TPE. In Fig. 2] we show the (normalized) integrand $I(\mu)$ in Eq. (2.24) as a function of $\mu$ for $r=\frac{1}{2} M_{\pi}^{-1}, r=M_{\pi}^{-1}$, $r=2 M_{\pi}^{-1}$ and for $\Lambda \rightarrow \infty$. For the LECs $c_{1,3}$ we use the values [15: $c_{1}=-0.81 \mathrm{GeV}^{-1}, c_{3}=-4.7 \mathrm{GeV}^{-1}$. As expected, at large distances $r \geq 2 M_{\pi}^{-1}$ the integral in Eq. (2.24) is dominated by low $-\mu$ components in the spectrum, where the chiral expansion for the spectral function is well behaved. However, at intermediate distances $r \sim 1 / M_{\pi} \sim 1.4 \mathrm{fm}$ the dominant contribution to the integral comes already from the region $\mu \sim 0.6 \mathrm{GeV}$, where only a very slow (if at all) convergence of the chiral expansion for $\rho(\mu)$ is expected. Certainly, at even shorter 


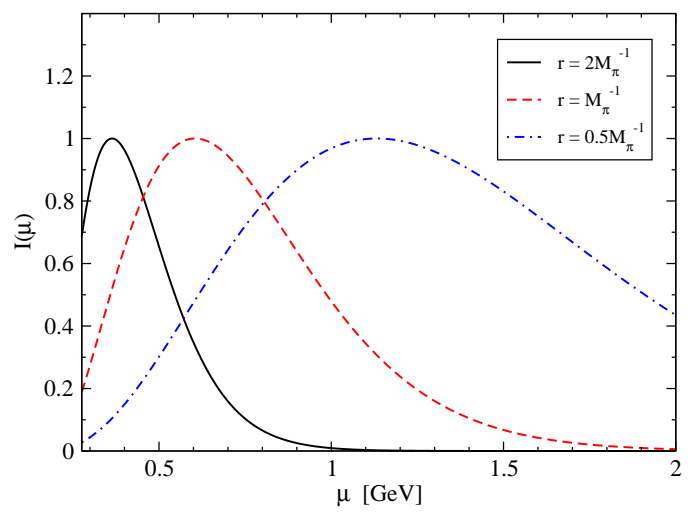

Fig. 2. The (normalized) integrand $I(\mu)$ in Eq. (2.24) for different distances $r$.

distances the resulting TPE potential is completely determined by the region of large $\mu$, where the spectral function is not properly described in chiral EFT. It is then clear that setting the upper limit of the integral in Eq. (2.24) to infinity, which corresponds to the DR result, leads to inclusion of spurious short-range physics in the TPE potential. We stress again that this problem solely arises because at the order we are working there are no contact terms which normally would absorb these contributions. On the other hand, introducing a finite cut-off $\Lambda$ in the spectral function representation according to Eq. (2.21) we explicitly exclude all short-range components (i.e. those ones with the range $R<\Lambda^{-1}$ ) from the TPE potential, which are still present in the DR expression. The procedure is legitimate and does not lead to any ambiguity if $\Lambda$ is chosen to be of the order of (or larger than) $\Lambda_{\chi} \sim M_{\rho}$, which provides a natural scale for the effective field theory. Various choices for $\Lambda$ lead to exactly the same result for lowenergy observables provided one keeps terms in all orders in the EFT expansion. Choosing a specific value for $\Lambda$ in the calculation at any finite order in the low-momentum expansion one implicitly makes a particular choice for the combination of the higher order contact terms. There are obviously no restrictions in choosing $\Lambda$ if all LECs are of the natural size and the expansion parameter in EFT is small. Both dimensional and cut-off regularizations lead to similar results and the unphysical short-range components of TPE resulting from keeping $\Lambda$ very large (or even $\infty$ ) are compensated by corresponding counter terms to the order at which one is working. In fact, as we will show in the next section, the NN potential at NLO may serve as an example of such a situation.

In certain cases, however, it appears to be advantageous to explicitly remove the spurious short-distance physics when calculating chiral loops. This happens, for instance, in $\mathrm{SU}(3)$ baryon chiral perturbation theory, where a much slower convergence of the chiral expansion is expected due to the relatively large mass of the strange quark. The leading nonanalytic components from loop corrections calculated using dimensional regularization in some cases seem to destroy the good agreement of the lowest order calcula- tion with data (like e.g. the Gell-Mann-Okubo relation for the baryon masses). Of course, this disagreement is corrected after inclusion of higher order contributions. However, the chiral expansion seems not to behave well and no clear convergence can be observed to the orders yet calculated (such a statement holds e.g. for the baryon masses [33 but not for the baryon magnetic moments [34,35]). Reformulating chiral EFT using a finite cut-off regularization allows to remove the spurious short-distance physics and to improve the convergence [26,27,28], if the cut-off procedure is implemented model-independently (as shown in Ref. [29]).

As discussed in the introduction, slow convergence of the chiral series for the $\mathrm{NN}$ interaction is observed if one uses the values for the LECs $c_{1,3,4}$ obtained in $\pi N$ scattering. The numerically large values of $c_{3,4}$ lead to a strong and attractive TPE at NNLO. Strong deviations from the NPSA results are observed in the $D$ - and $F$-waves which are parameter-free at this order and are still sensitive to the TPE contribution. We will demonstrate in the next section that the problems with the convergence are not due to the large values of $c_{3,4}$ which provide a proper longrange part of TPE [23 24], but rather due to unphysical short-range components in the DR expressions for the potential, which can (and should) be avoided using the CR.

To close this section we will give analytical expressions for TPE in the coordinate space. For the NNLO contributions one finds:

$$
\begin{aligned}
\tilde{V}_{C}^{\Lambda}(r)= & \frac{3 g_{A}^{2}}{32 \pi^{2} F_{\pi}^{4}} \frac{e^{-2 x}}{r^{6}}\left[2 c_{1} x^{2}\left(1+x^{2}\right)^{2}\right. \\
& \left.+c_{3}\left(6+12 x+10 x^{2}+4 x^{3}+x^{4}\right)\right] \\
& -\frac{3 g_{A}^{2}}{128 \pi^{2} F_{\pi}^{4}} \frac{e^{-y}}{r^{6}}\left[4 c_{1} x^{2}\left(2+y(2+y)-2 x^{2}\right)\right. \\
& +c_{3}\left(24+y\left(24+12 y+4 y^{2}+y^{3}\right)\right. \\
& \left.\left.-4 x^{2}\left(2+2 y+y^{2}\right)+4 x^{4}\right)\right] \\
\tilde{W}_{T}^{\Lambda}(r)=- & \frac{g_{A}^{2}}{48 \pi^{2} F_{\pi}^{4}} \frac{e^{-2 x}}{r^{6}} c_{4}(1+x)\left(3+3 x+x^{2}\right) \\
& +\frac{g_{A}^{2}}{768 \pi^{2} F_{\pi}^{4}} \frac{e^{-y}}{r^{6}} c_{4}\left(48+48 y+24 y^{2}+7 y^{3}+y^{4}\right. \\
& \left.-4 x^{2}\left(8+5 y+y^{2}\right)\right) \\
\tilde{W}_{S}^{\Lambda}(r)= & \frac{g_{A}^{2}}{48 \pi^{2} F_{\pi}^{4}} \frac{e^{-2 x}}{r^{6}} c_{4}(1+x)\left(3+3 x+2 x^{2}\right) \\
& -\frac{g_{A}^{2}}{384 \pi^{2} F_{\pi}^{4}} \frac{e^{-y}}{r^{6}} c_{4}\left(24+24 y+12 y^{2}+4 y^{3}+y^{4}\right. \\
& \left.-4 x^{2}\left(2+2 y+y^{2}\right)\right)
\end{aligned}
$$

with the abbreviations $x=M_{\pi} r, y=\Lambda r$. For the NLO contributions we could not perform integrations in Eqs. (2.24)(2.26) analytically. In the chiral limit $\left(M_{\pi}=0\right)$ the results 
take, however, the following simple form:

$$
\begin{aligned}
\left.\tilde{W}_{C}^{\Lambda}(r)\right|_{M_{\pi}=0}= & \frac{23 g_{A}^{4}-10 g_{A}^{2}-1}{1536 \pi^{3} F_{\pi}^{4} r^{5}}[-6 \\
& \left.+e^{-y}\left(6+6 y+3 y^{2}+y^{3}\right)\right] \\
\left.\tilde{V}_{T}^{\Lambda}(r)\right|_{M_{\pi}=0}= & \frac{g_{A}^{4}}{256 \pi^{3} F_{\pi}^{4} r^{5}}[-15 \\
& \left.+e^{-y}\left(15+15 y+6 y^{2}+y^{3}\right)\right] \\
\left.\tilde{V}_{S}^{\Lambda}(r)\right|_{M_{\pi}=0}= & \frac{g_{A}^{4}}{128 \pi^{3} F_{\pi}^{4} r^{5}}[6 \\
& \left.-e^{-y}\left(6+6 y+3 y^{2}+y^{3}\right)\right]
\end{aligned}
$$

Certainly, the large-distance asymptotical behavior of the potential is unaffected by the cut-off procedure provided that $\Lambda \gg M_{\pi}$. In Fig. [3 we compare the isoscalar central part of TPE obtained using CR and DR. The strongest effects of the cut-off are observed at intermediate $\left(\sim M_{\pi}^{-1}\right)$ and smaller distances, where TPE becomes unphysically attractive if DR is used. In contrast, removing the large components in the mass spectrum of the TPE with the reasonably chosen cut-off $\Lambda=500 \ldots 800 \mathrm{MeV}$ greatly reduces this unphysical attraction and the resulting potential is of the same order in magnitude as the one obtained in phenomenological boson-exchange models. It remains to say that the CR expressions for the TPE are still not regular in the origin, although the short-distance behavior is milder than in the case of DR and the leading singularities at $r=0$ are removed. For example, while $\tilde{V}_{C}(r) \propto 1 / r^{6}, \tilde{V}_{C}^{\Lambda}(r) \propto 1 / r^{5}$ for $r \rightarrow 0$. The Schrödinger (or LS) equation has still to be regularized by introduction of an additional cut-off.

\section{Chiral TPE at NNLO and peripheral NN scattering}

In this section we will apply the OPE and TPE nuclear force at NNLO, calculated using the cut-off regularization as described in the previous section, to NN phase shifts with orbital angular momentum $l \geq 2$ and to mixing angles with $j \geq 2$. As already pointed out in the introduction, no contact terms contribute at NNLO to the scattering amplitude in these channels. Consequently, such peripheral phase shifts are entirely determined by the longrange part of the nuclear force and thus provide a sensitive test of the chiral TPE. The OPE potential at NLO (and NNLO) is given by

$$
V_{\mathrm{OPE}}=-\frac{g_{A}^{2}}{4 F_{\pi}^{2}}\left(1-\frac{4 M_{\pi}^{2}}{g_{A}} d_{18}\right) \boldsymbol{\tau}_{1} \cdot \boldsymbol{\tau}_{2} \frac{\left(\sigma_{1} \cdot \mathbf{q}\right)\left(\sigma_{2} \cdot \mathbf{q}\right)}{q^{2}+M_{\pi}^{2}}
$$

where the LEC $d_{18}$ is related to the Goldberger-Treiman discrepancy via

$$
\frac{g_{\pi N}}{m}=\frac{g_{A}}{F_{\pi}}\left(1-\frac{2 M_{\pi}^{2}}{g_{A}} d_{18}\right) .
$$

In what follows we use $g_{A}=1.26, F_{\pi}=92.4 \mathrm{MeV}, d_{18}=$ $-0.97 \mathrm{GeV}^{-2}$, which leads to $g_{\pi N} \simeq 13.2$. For TPE at NLO and NNLO we use Eqs. (2.4), (2.8) with the functions $L(q), A(q)$ replaced by $L^{\Lambda}(q), A^{\Lambda}(q)$ defined in Eq. (2.22). For the $c_{i}$ 's we adopt the values [15]: $c_{1}=-0.81 \mathrm{GeV}^{-1}$, $c_{3}=-4.70 \mathrm{GeV}^{-1}, c_{4}=3.40 \mathrm{GeV}^{-1}$.

The explicit expressions for the partial-wave decomposition of the potential can be found in Ref. 21. The partial-wave projected Lippmann-Schwinger (LS) equation for the $\mathrm{NN} \mathrm{T}-$ matrix reads:

$$
\begin{aligned}
T_{l, l^{\prime}}^{s j}\left(p^{\prime}, p\right)= & V_{l, l^{\prime}}^{s j}\left(p^{\prime}, p\right)+\sum_{l^{\prime \prime}} \int \frac{d^{3} p^{\prime \prime}}{(2 \pi)^{3}} V_{l, l^{\prime \prime}}^{s j}\left(p^{\prime}, p^{\prime \prime}\right) \\
& \times \frac{m}{p^{2}-\left(p^{\prime \prime}\right)^{2}+i \epsilon} T_{l^{\prime \prime}, l^{\prime}}^{s j}\left(p^{\prime \prime}, p\right),
\end{aligned}
$$

where the on-shell S- and T-matrices are related via

$$
S_{l, l^{\prime}}^{s j}(p, p)=\delta_{l l^{\prime}} 1^{s j}-\frac{i}{8 \pi^{2}} p m T_{l, l^{\prime}}^{s j}(p, p) .
$$

Since the chiral potential grows at large momenta, the LS equation (3.3) has to be regularized, ${ }^{2}$ which requires introduction of an additional cut-off (which can be done consistently with the CR discussed so far). It is commonly believed (and observed in various boson-exchange and phenomenological potential models, see also Ref. [18] that because of the centrifugal barrier the $\mathrm{NN}$ interaction in the peripheral partial waves becomes weak enough to be treated perturbatively. This is also confirmed by the smallness of the corresponding phase shifts. To calculate phase shifts in high partial waves one may therefore use the Born approximation to the $\mathrm{T}$-matrix: ${ }^{3}$

$$
T_{l, l^{\prime}}^{s j}\left(p^{\prime}, p\right) \sim V_{l, l^{\prime}}^{s j}\left(p^{\prime}, p\right) .
$$

Such a procedure, which is analogous to the one of Refs. 9, 38, allows to avoid the introduction of an additional cutoff in the LS equation and will be adopted in the present work. One should, however, always keep in mind that this approximation breaks down if the phase shifts become large.

Before presenting our predictions for the high partial waves, we would like to specify the differences between our analysis and the one of Kaiser et al. 9]:

- First of all, we strictly follow the lines of Weinberg's power counting and do not include relativistic corrections to the nuclear force at NNLO, which have been included in Ref. 9]. In contrast to this reference, we use non-relativistic kinematics when calculating phase shifts.

- We also do not include the contribution from once iterated OPE, which turns out to be numerically small

\footnotetext{
2 This regularization of the LS equation should not be confused with the $\mathrm{CR}$ of the chiral loops discussed in previous sections.

3 Notice, however, that the weakness of the NN interaction for high values of $l$ is not related to the chiral expansion and does not follow from the power counting.
} 

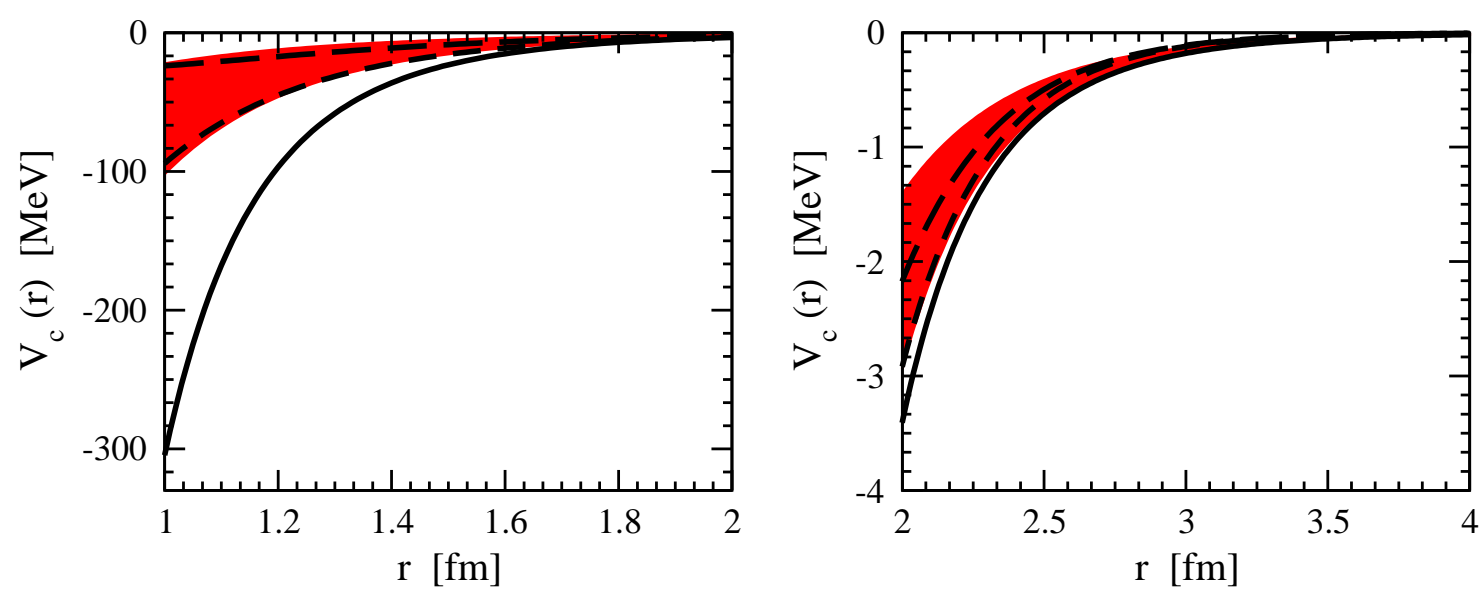

Fig. 3. The isoscalar central TPE potential at NNLO in $r$-space. The solid line shows the DR result corresponding to $\Lambda=\infty$ while the red band results from varying $\Lambda$ between 500 and $800 \mathrm{MeV}$. The short(long-) dashed line shows the phenomenological $\sigma(\sigma+\omega+\rho)$ contributions based on the isospin triplet configuration space version (OBEPR) of the Bonn potential [36].

in most channels. From the point of view of the power counting, there is no reason to include once iterated OPE and not to include two-, three-, ... times iterated OPE.

- We use slightly different (and more modern) values for the LECs $c_{1,3,4}$ and for $g_{A}$.

- The last and most important difference is that we use the finite cut-off $\Lambda$ to regularize chiral loop integrals. This is in strong contrast with the analysis of Ref. 9, where DR corresponding to $\Lambda=\infty$ has been adopted. As it is clear from the above discussion, optimal values for the cut-off are those close to the scale where the EFT description becomes inaccurate. Taking a too small $\Lambda$ will remove the truly long-distance physics while too large values for the cut-off may affect the convergence of the EFT expansion due to inclusion of spurious short-distance physics. We will therefore vary $\Lambda$ in the range $\Lambda=500 \ldots 800 \mathrm{MeV}$ which appears to be physically reasonable and matches well with both $M_{\rho}$ and the cut-off used in the LS equation for NN scattering [18].

Let us start with the D-waves which are shown in Fig. 4 The LO result represented by pure OPE already provides a good approximation to the phase shifts in the ${ }^{3} D_{1}$ and ${ }^{3} D_{2}$ partial waves and to the mixing angle $\epsilon_{2}$. It is too weak in the ${ }^{1} D_{2}$ channel and does not describe properly the ${ }^{3} D_{3}$ phase shift. The letter appears to be quite small $\left(|\delta| \sim 4.6^{\circ}\right.$ at $\left.E_{\text {lab }}=300 \mathrm{MeV}\right)$ compared to the other $\mathrm{D}$-wave phase shifts $\left(|\delta| \sim 9.7^{\circ} \ldots 25.5^{\circ}\right)$. The reason is that partial-wave projected OPE, taken on the energy shell, is strongly suppressed in this channel. Consequently, the ${ }^{3} D_{3}$ phase shift is quite sensitive to TPE but also to the iteration of the potential which we neglect in the present analysis.
The NLO predictions obtained using dimensional regularization are shown by the dashed curves. One observes a visible improvement for the ${ }^{1} D_{2}$ phase shift and for $\epsilon_{2}$ while the NLO corrections go in the wrong direction in the ${ }^{3} D_{1}$ and ${ }^{3} D_{3}$ channels. The NNLO predictions calculated with dimensional regularization are depicted by the solid lines. The good agreement with the data observed at LO and NLO is destroyed in all partial waves for energies $E_{\text {lab }}>50 \mathrm{MeV}$ and the chiral expansion does not seem to converge. Note that at $\mathrm{N}^{3} \mathrm{LO}$ one independent contact operator contributes to each $\mathrm{D}$-wave so that the agreement with the data will presumably be restored, see also Ref. 21] for a related discussion. Note that the results presented here are parameter-free and are very similar to the ones of Ref. 9. No breakdown of the chiral expansion at NNLO is observed using the CR in the chiral loops and choosing $\Lambda=500 \ldots 800 \mathrm{MeV}$. This proves explicitly that strong disagreement with the data resulting from chiral TPE at NNLO is due to unphysical short-distance components, which are kept in the DR expressions for the potential. The use of a momentum space cut-off keeps only the long-distance portion of the chiral loops and leads to a greatly improved behavior. Our predictions agree with the data in the ${ }^{1} D_{2}$ and ${ }^{3} D_{1}$ channels and go into the right direction in the ${ }^{3} D_{3}$ partial wave and for $\epsilon_{2}$. Note that the uncertainty due to the variation of $\Lambda$ is quite significant at higher energies. The NLO bands are narrower than the NNLO ones as a consequence of the fact that the leading TPE contribution is numerically quite small.

Our predictions for the $\mathrm{F}$-wave phase shifts and for $\epsilon_{3}$ are presented in Fig. 5 Although the situation with DR subleading TPE is not as dramatic as for D-waves, a too strong attraction is clearly visible in the ${ }^{3} F_{2},{ }^{3} F_{3}$ and ${ }^{3} F_{4}$ partial waves. Removing the short-distance components of TPE with the cut-off regularization leads to a significant 

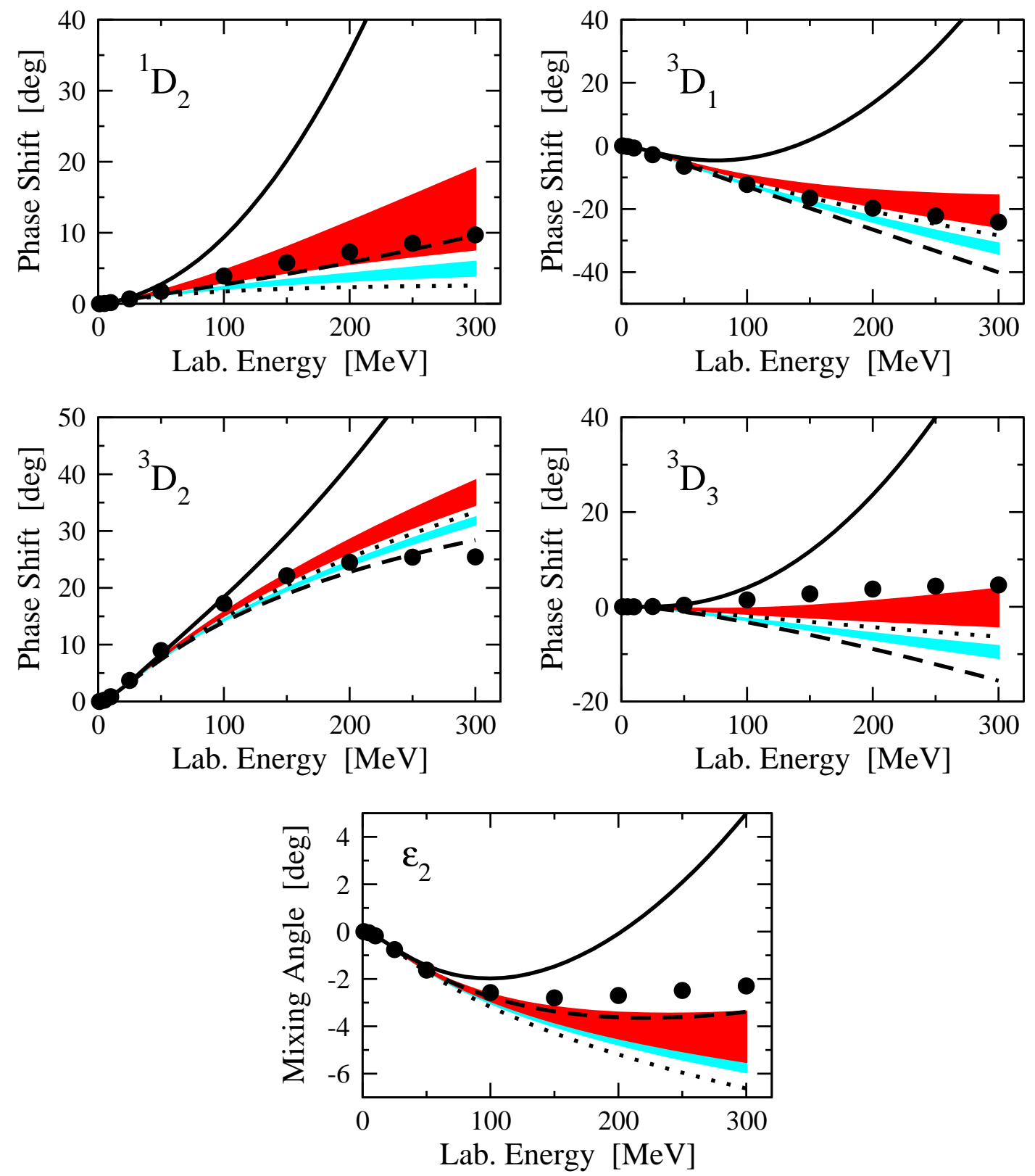

Fig. 4. D-wave NN phase shifts and mixing angle $\epsilon_{2}$ versus the nucleon laboratory energy. The dotted curve is the LO result (i.e. pure OPE), while the dashed (solid) curve refers to NLO (NNLO) results for OPE+TPE with the potential calculated using dimensional regularization. The light (dark) shaded band shows the NLO (NNLO) predictions with chiral TPE obtained using the cut-off regularization with $\Lambda=500 \ldots 800 \mathrm{MeV}$. The filled circles depict the Nijmegen PSA results [37.

improvement in the ${ }^{3} F_{3}$ and ${ }^{3} F_{4}$ channels, while additional repulsion is still missing in the ${ }^{3} F_{2}$ partial wave.

For the G- and higher partial waves we observe very similar results for both DR and CR TPE and the phase shifts are essentially given by OPE. Notice that additional attraction is generated from iterated OPE in the ${ }^{3} G_{5}$ partial wave [9], which is not included in the present work. Therefore, the strong disagreement with the data in this channel is presumably just an artefact of the Bornapproximation.

Finally, we would like to point out that the typical uncertainty of $\sim 30 \%$ observed in our NNLO predictions at $E_{\text {lab }}=300 \mathrm{MeV}$ is consistent with the power counting. Indeed, the $\mathrm{N}^{3} \mathrm{LO}$ counter terms are expected to provide corrections to the S-matrix of the order $Q^{4} / \Lambda_{\chi}^{4}$. Taking $Q \sim 375 \mathrm{MeV}$, which corresponds to $E_{\text {lab }}=300 \mathrm{MeV}$, 

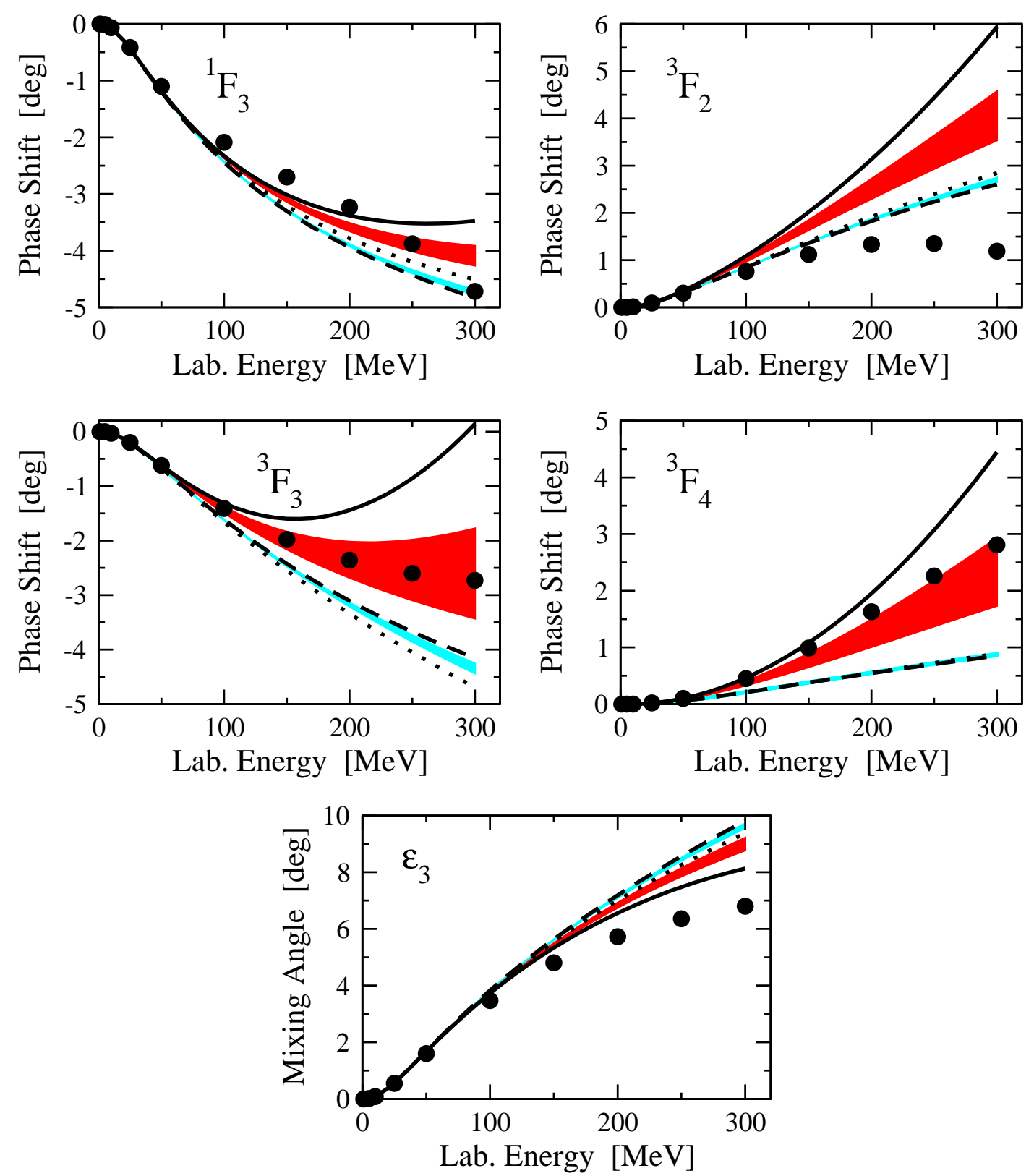

Fig. 5. F-wave NN phase shifts and mixing angle $\epsilon_{3}$ versus the nucleon laboratory energy. For notation see Fig. 4

and identifying $\Lambda_{\chi}$ with the smallest value used for the cut-off $\Lambda$, i.e. $\Lambda=500 \mathrm{MeV}$, one estimates the $\mathrm{N}^{3} \mathrm{LO}$ effects as $\sim 32 \%$. As already stated before, the uncertainty is larger in the cases, where phase shifts are numerically small (like, for instance, in the ${ }^{1} D_{2},{ }^{3} D_{3},{ }^{3} G_{5}$ partial waves). Although the following remark is quite obvious, we stress that a two-nucleon potential based on a systematic EFT approach should not be fine-tuned to fulfill a $\chi^{2} /$ dof $\simeq 1$ as it is done in more conventional approaches. This does, however, not mean that such a precision can not be reached.

\section{Summary and conclusions}

In this paper we have considered the two-nucleon potential in chiral effective field theory, making use of a novel method of regularizing the pion loop integrals. For that, we have considered the spectral functions obtained from the NLO and NNLO TPE contributions and argued that only masses below the chiral symmetry breaking scale should contribute in the loop integrals. This can be easily implemented by applying a cut-off to the spectral functions. Varying the cut-off between 500 and $800 \mathrm{MeV}$ (as 

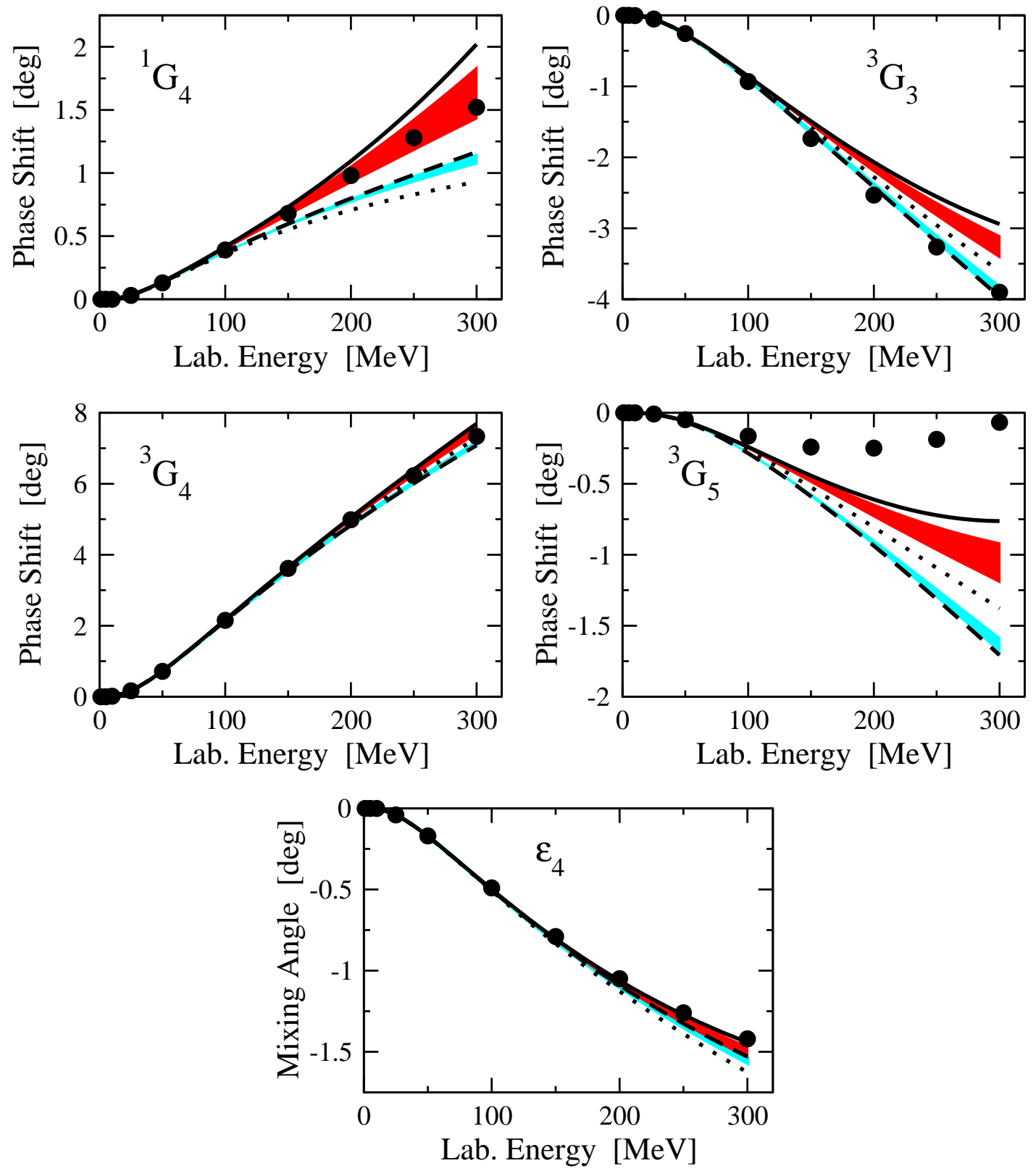

Fig. 6. G-wave NN phase shifts and mixing angle $\epsilon_{4}$ versus the nucleon laboratory energy. For notation see Fig. 4

given by the mass of the heaviest Goldstone boson, the eta, and the mass of the lightest resonance, the rho), we find the following pertinent results:

1) From the regularized spectral functions, we have constructed the coordinate space representations of the various components of the NN potential. The isoscalar central TPE shown in Fig. 3 agrees with phenomenological potentials. The strong unphysical attraction of the TPE is a short-range phenomenon which is suppressed in CR by choosing $\Lambda \leq M_{\rho}$, whereas in the Bonn potential $\pi \rho$ exchange cancels the strong attrac- tive TPE, which is one particular model for this kind of short-range physics.

2) We have considered the peripheral partial waves $(l \geq$ 2 ), because at NNLO, these are given entirely by OPE and TPE with no free parameters. We have calculated these phases in Born approximation which should be legitimate at least for the $\mathrm{D}$ - and higher waves, see Ref. 18 for more discussion. The uncertainty in most $\mathrm{D}$ - and $\mathrm{F}$-waves at NNLO is still sizeable even with the finite cut-off. This has to be expected because of the large values of $c_{i}$ 's. The results for $\mathrm{D}$ - and $\mathrm{F}$-waves 
are still not completely converged at NNLO, but the error of about $10(1)^{\circ}$ at $E_{\text {lab }}=300 \mathrm{MeV}$ for the D$(\mathrm{F}-)$ waves appears reasonable. There is no breakdown of the chiral expansion for $\mathrm{D}$-waves beyond $T_{\text {lab }}=50$ $\mathrm{MeV}$ and for $\mathrm{F}$-waves beyond $T_{\mathrm{lab}}=150 \mathrm{MeV}$ as found in Ref. 9] using dimensional regularization.

3) It is no surprise that the NNLO TPE gives larger corrections than the NLO one because of the delta contributions subsumed in the LECs $c_{3}$ and $c_{4}$. One might therefore contemplate including the $\Delta(1232)$ explicitly in the effective Lagrangian (see e.g. 7]), because in such a theory most (but not all!) of the NNLO effects are shifted to NLO, provided that a systematic analysis of pion-nucleon scattering in such a scheme is available (for attempts see e.g. 39,40 41). However, for obtaining a precise potential one still would have to go to NNLO, which is considerably more complicated than in the pion-nucleon EFT, as witnessed by the fact that no complete fourth order calculation with deltas in the single nucleon sector exists.

4) We stress that dimensional regularization is by no means ruled out by such considerations. In general, for quickly converging expansions, it should be the method of choice. If, however, the convergence for some well understood physical reason is slow and (some) observables become sensitive to spurious short-distance physics kept in $\mathrm{DR}$, it might be preferable to use CR, as done here. In our case the LECs $c_{3,4}$ are large. Choosing DR, one generates a series of higher order contact interactions, which are $\propto c_{3,4}$ and therefore large. The $\mathrm{D}$ - and $\mathrm{F}$ waves are certainly most sensitive to such contact interactions and are thus strongly affected.

5) The cut-off used here is not a form-factor and is not related to the finite extension of the nucleon. Obviously, the precise shape of the regulating function and the precise value of $\Lambda$ are not important (as long as the value for $\Lambda$ is well above the two-pion threshold, $\Lambda>2 M_{\pi}$ and below the scale of chiral symmetry breaking, $\left.\Lambda<\Lambda_{\chi}\right)$.

In a subsequent publication, we will apply CR to the low partial waves in the non-perturbative regime, where we have to solve the regularized Lippmann-Schwinger equation Eq. (3.3) to generate the bound and scattering states. It can be demonstrated that there are no deeply bound states and low-energy observables are not affected by CR.

\section{Acknowledgments}

We would like to thank John Donoghue for important comments and Norbert Kaiser and Jambul Gegelia for useful discussions. This work is supported in part by the Deutsche Forschungsgemeinschaft (E.E.).

\section{References}

1. U.-G. Meißner, Rept. Prog. Phys. 56 (1993) 903.

2. A. Pich, Rept. Prog. Phys. 58 (1995) 563.
3. V. Bernard, N. Kaiser and U.-G. Meißner, Int. J. Mod. Phys. E 4 (1995) 193.

4. G. Ecker, Prog. Part. Nucl. Phys. 36 (1996) 71.

5. S. Weinberg, Phys. Lett. B 251 (1990) 288.

6. S. Weinberg, Nucl. Phys. B 363 (1991) 3.

7. C. Ordóñez, L. Ray and U. van Kolck, Phys. Rev. C 53 (1996) 2086.

8. J.L. Friar, S.A. Coon, Phys. Rev. C 49 (1994) 1272.

9. N. Kaiser, R. Brockmann, and W. Weise, Nucl. Phys. A 625 (1997) 758.

10. E. Epelbaoum, W. Glöckle, and U.-G. Meißner, Nucl. Phys. A 637 (1998) 107.

11. V. Bernard, N. Kaiser, and U.-G. Meißner, Nucl. Phys. B 457 (1995) 147.

12. V. Bernard, N. Kaiser, and U.-G. Meißner, Nucl Phys. A 615 (1997) 483.

13. M. Mojžiš, Eur. Phys. J. C2 (1998) 181.

14. N. Fettes, U.-G. Meißner, and S. Steininger, Nucl. Phys. A 640 (1998) 199.

15. P. Büttiker, and U.-G. Meißner, Nucl. Phys. A 668 (2000) 97.

16. N. Fettes and U.-G. Meißner, Nucl. Phys. A 676 (2000) 311.

17. N. Fettes and U.-G. Meißner, Nucl. Phys. A 693 (2001) 693.

18. E. Epelbaum, A. Nogga, W. Glöckle, H. Kamada, U.G. Meißner and H. Witala, Eur. Phys. J. A 15 (2002) 543.

19. A. D. Galanin, A. F. Grashin, B. L. Ioffe and I. Ya. Pomeranchuk, Nucl. Phys. 17 (1960) 181.

20. V.G.J. Stoks, R.A.M. Klomp, M.C.M. Rentmeester, and J.J. de Swart, Phys. Rev. C 48 (1993) 792.

21. E. Epelbaum, W. Glöckle, and U.-G. Meißner, Nucl. Phys. A 671 (2000) 295.

22. S.R. Beane, V. Bernard, E. Epelbaum, U.-G. Meißner and D. R. Phillips, accepted for publication in Nucl. Phys. A.

23. M.C.M. Rentmeester, R.G.E. Timmermans, J.L. Friar, and J.J. de Swart, Phys. Rev. Lett. 82 (1999) 4992.

24. M.C.M. Rentmeester, R.G.E. Timmermans, and J.J. de Swart, accepted for publication in Phys. Rev. C.

25. J. Gasser, Annals Phys. 136 (1981) 62.

26. J.F. Donoghue, and B.R. Holstein, Phys. Lett. B 436 (1998) 331 .

27. J.F. Donoghue, B.R. Holstein, and B. Borasoy, Phys. Rev. D 59 (1999) 036002.

28. B. Borasoy, B.R. Holstein, R. Lewis, and P.-P.A. Ouimet, Phys. Rev. D 66 (2002) 094020.

29. V. Bernard, T.R. Hemmert and U.-G. Meißner, "Cutoff schemes in chiral perturbation theory", in preparation.

30. J.L. Friar, Mod. Phys. Lett. A11 (1996) 3043.

31. M. Chemtob, J.W. Durso, and D.O. Riska, Nucl. Phys. B 38 (1972) 141.

32. E. Epelbaum, U.-G. Meißner, W. Glöckle, Nucl.Phys. A714 (2003) 535 .

33. B. Borasoy and U.-G. Meißner, Annals Phys. 254 (1997) 192.

34. U.-G. Meißner and S. Steininger, Nucl. Phys. B 499 (1997) 349.

35. B. Kubis and U.-G. Meißner, Eur. Phys. J. C 18 (2001) 747.

36. R. Machleidt, K. Holinde and Ch. Elster, Phys. Rep. 149 (1987) 1.

37. V.G.J. Stoks, R.A.M. Klomp, M.C.M. Rentmeester, and J.J. de Swart, Phys. Rev. C 48 (1993) 792. 
38. D.R. Entem, and R. Machleidt, Phys. Rev. C 66 (2002) 014002.

39. P. J. Ellis and H. B. Tang, Phys. Rev. C 56 (1997) 3363.

40. P. J. Ellis and H. B. Tang, Phys. Rev. C 57 (1998) 3356.

41. N. Fettes and U.-G. Meißner, Nucl. Phys. A 679 (2001) 629. 